\title{
Vasculitis Around the World: Epidemiologic Insights into Causality and a Need for Global Partnerships
}
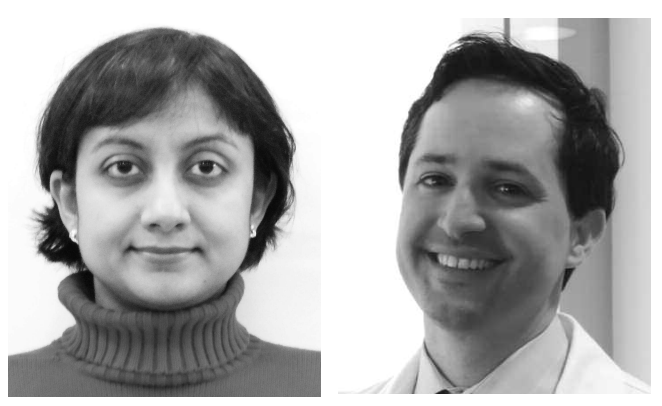

Vasculitis is a family of rare diseases characterized by inflammation of blood vessels. Causal factors for most forms of vasculitis have not been identified. Certain forms of vasculitis are more prevalent in specific parts of the world, and clinical features of a particular type of vasculitis can vary by region. Observed geographic differences in incidence and prevalence of various forms of vasculitis and corresponding phenotypic diversities in different parts of the world suggest that epidemiological studies may generate important insights that can be leveraged to identify the causes of vasculitis.

Geographic differences in incidence rates have been observed in the major forms of small and large vessel vasculitis (Figure 1). Takayasu arteritis (TA), a prototypical form of large vessel vasculitis (LVV), is more common in Japan compared to the United States and Europe. In contrast, giant cell arteritis (GCA), the other major form of LVV, is relatively uncommon in Japan compared to Europe and North America ${ }^{1}$. Incidence of antineutrophil cytoplasmic antibody (ANCA)-associated vasculitis (AAV), the prototypical form of small vessel vasculitis, also differs between Asian and Western countries. Of the 2 major forms of AAV, a higher incidence of granulomatosis with polyangiitis (GPA) has been reported in Europe and the United States compared to Japan, and a higher incidence of microscopic polyangiitis (MPA) has been reported in Japan and China compared to the United Kingdom and the United States ${ }^{2}$.

Beyond differences in incidence rates, regional differences in clinical features within a specific form of vasculitis have been reported. In Japan, TA is typically a disease of young women with predominant aortic arch involvement ${ }^{3}$. Ophthalmic circulatory disturbances, ischemic heart disease, arrhythmia, congestive heart failure, and cerebral ischemia are commonly reported ${ }^{1}$. Similar clinical features of disease and distributions of arterial pathology are observed in patients with TA from the United States and Western Europe ${ }^{4,5}$. In contrast, middle-aged men in China or India can develop TA typically involving the abdominal aorta and renal arteries with corresponding symptoms of lower extremity claudication and renovascular hypertension ${ }^{3,6}$.

Regional differences in features of AAV have also been demonstrated. Compared to patients from the United Kingdom, Japanese patients with MPA are more likely to be myeloperoxidase (MPO)-ANCA positive and older at the time of diagnosis, with a higher prevalence of interstitial pneumonia, pulmonary fibrosis, and peripheral neuropathy ${ }^{7}$. In contrast, a higher serum creatinine at time of diagnosis, cutaneous vasculitis, and ocular manifestations are more commonly observed in patients with MPA from the United Kingdom ${ }^{7}$.

In the current issue of The Journal, Furuta, et al report findings that add to our understanding of regional diversity in $\mathrm{AAV}^{8}$. While much has been made of the high incidence of MPA in Japan, the present study is unique in its attempt to characterize GPA in a cohort of Japanese patients in comparison to a cohort from Europe ${ }^{8}$. Compared to patients with GPA from the UK, Japanese patients with GPA were older at the time of diagnosis and were more likely to have ocular, mucosal, and pulmonary manifestations. Ear, nose, and throat involvement was observed in $>80 \%$ of patients in both the UK and Japanese cohorts. Among pulmonary manifestations, fibrosis was more common in Japan whereas pulmonary hemorrhage was more prevalent in the UK cohort, a pattern of respiratory involvement that mirrors what has been previously reported in patients with MPA from these countries. Similarly to MPA, patients with GPA from the UK also had higher serum creatinine at the time of diagnosis compared to their Japanese counterparts ${ }^{7,8}$. A summary of findings for the 2 studies by Furuta, et al that compared regional diversity in AAV in Japan versus the UK is presented in Table 1 .

The current study by Furuta, et al also adds to our understanding of rather striking regional differences in ANCA serotype between Japan and the Western world ${ }^{8}$. Within patients classified as GPA, proteinase 3 (PR3)-ANCA

See GPA in UK versus Japanese cohorts, page 216 


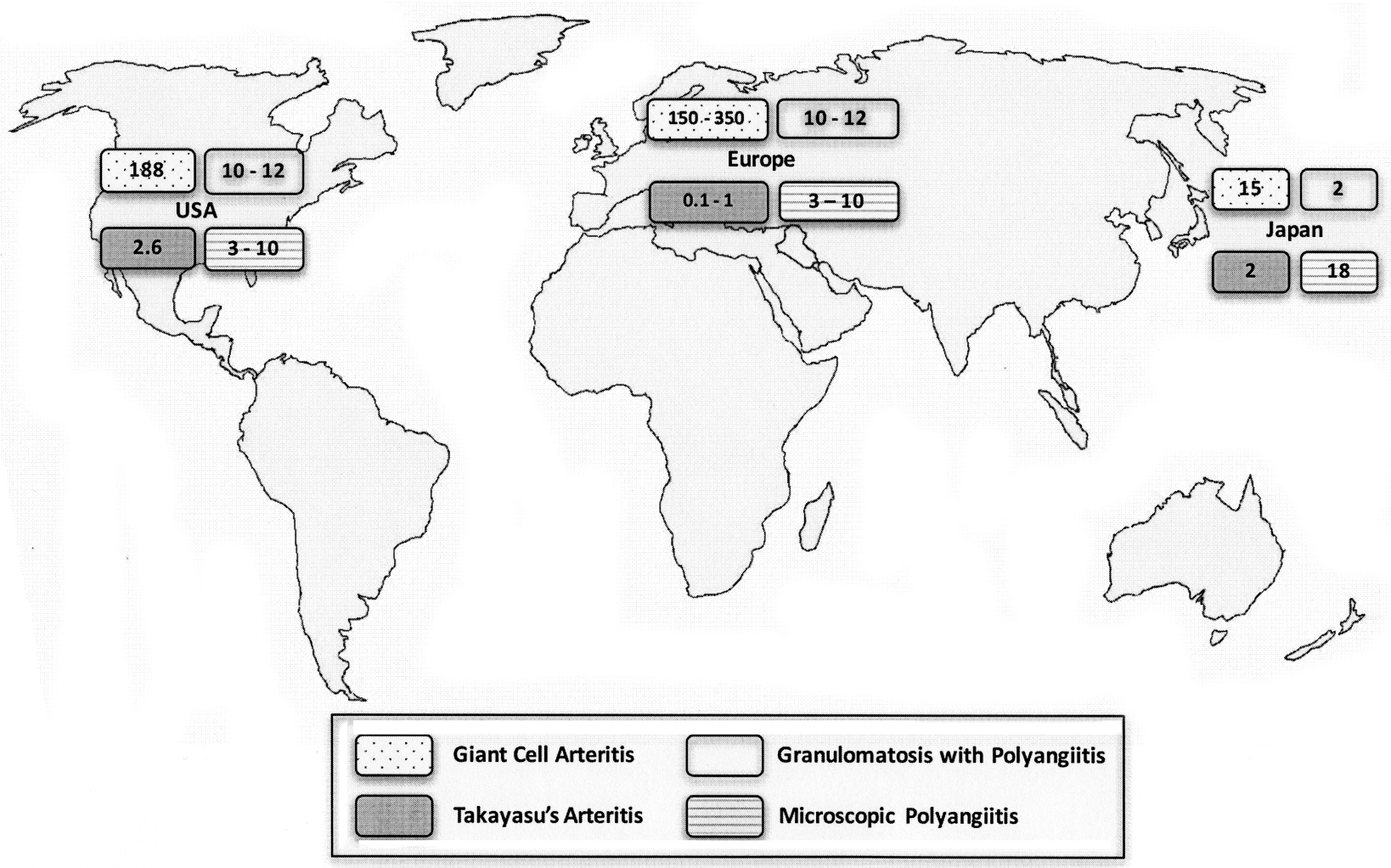

Figure 1. Annual incidence of different forms of vasculitis in the United States, Europe, and Japan (per million population). A higher incidence of giant cell arteritis and granulomatosis with polyangiitis has been reported in northern regions of the world. Data are lacking from Africa, South America, and many parts of Asia.

Table 1. Regional differences in clinical features of ANCA-associated vasculitis comparing patients from Japan and the United Kingdom ${ }^{7,8}$.

Regional Differences Common to Both GPA and MPA

Regional Differences Specific to GPA or MPA

\begin{tabular}{|c|c|c|c|c|c|c|}
\hline Clinical Feature & Japan & UK & Clinical Feature & AAV Subtype & Japan & UK \\
\hline Respiratory involvement & More frequent & Less frequent & & MPA & Less frequent & More frequent \\
\hline $\begin{array}{l}\text { Serum creatinine at } \\
\text { diagnosis }\end{array}$ & Lower & Higher & & MPA & \multicolumn{2}{|c|}{ No difference } \\
\hline MPO-ANCA & More frequent & Less frequent & Cutaneous & GPA & \multicolumn{2}{|c|}{ No difference } \\
\hline $\begin{array}{l}\text { Cumulative } \\
\text { cyclophosphamide }\end{array}$ & Less & More & & MPA & \multicolumn{2}{|c|}{ No difference } \\
\hline Plasma exchange & Less frequent & More frequent & Peripheral nervous & GPA & \multicolumn{2}{|c|}{ No difference } \\
\hline & & & system & MPA & More frequent & Less frequent \\
\hline Biologic therapy & Less frequent & More frequent & & & & \\
\hline
\end{tabular}

ANCA: antineutrophil cytoplasmic antibodies; GPA: granulomatosis with polyangiitis; MPA: microscopic polyangiitis; AAV: ANCA-associated vasculitis; MPO: myeloperoxidase; PR3: proteinase 3.

positivity was more common in the UK cohort compared to the Japanese cohort ( $85 \%$ vs $61 \%)$. However, prevalence of MPO-ANCA was significantly higher in Japanese versus UK patients diagnosed with MPA $(96 \% \text { vs } 69 \%)^{7}$. Given the increased awareness of differences in genetic associations and clinical outcomes in patients with MPO-ANCA versus

$$
\text { Personal non-commercial use only. The Journal of Rheumatology Copyright (c) 2017. All rights reserved. }
$$


PR3-ANCA ${ }^{9,10}$, accompanying studies are needed to define biologic factors that potentially underlie differences in the prevalence of ANCA serotypes in specific parts of the world.

In addition to intercontinental differences in disease incidence, latitude may also contribute to geographic diversity. A trend of increasing incidence of GCA at higher latitudes in the northern hemisphere has been observed ${ }^{11,12,13}$. Significantly lower incidence of GCA was reported in southern parts of the United States (1.58/100,000 population $>50 \mathrm{yrs}$ ) compared to more northern regions of the country $(11.7 / 100,000 \text { population }>50 \mathrm{yrs})^{13}$. Similarly, incidence of GCA is lower in Southern European and Mediterranean countries compared to Northern European and Scandinavian countries ${ }^{11}$. Some European studies from Spain and Norway failed to demonstrate latitudinal gradient effect on the incidence of $\mathrm{GCA}^{14}$. GPA is also more common in northern versus southern Europe ${ }^{7}$. Immunomodulatory effects of vitamin D and ultraviolet radiation have been proposed as possible explanations for this phenomenon ${ }^{15}$. A similar north-south latitudinal gradient has been observed but not explained in other immune-mediated diseases, including multiple sclerosis ${ }^{15}$.

Various potential causes may explain geographic diversity in vasculitis, including the contributions of environmental exposures, infections, and genetic susceptibility. A higher incidence of GPA has been reported in rural areas; however, studies on farming and incidence of GPA have shown mixed results ${ }^{14,15}$. Silica exposure has been implicated as a possible cause of $\mathrm{AAV}^{8}$. Studies on exposure to inhaled fumes and particulates, pesticides, and hydrocarbons and AAV prevalence have yielded conflicting results ${ }^{10}$. Infectious processes have long been postulated as causal factors in vasculitis, and global differences in infection rates could contribute to geographic diversity. Future studies that assess the microbiome in association with dietary practices, genetic factors, and clinical data could reveal causes that shape patterns of geographic diversity observed in vasculitis.

Ethnic and genetic diversity may account for differences in disease susceptibility and thereby influence the geographic distribution and phenotypic variations of vasculitis. A significantly higher prevalence of GCA has been reported in whites compared to African Americans and Asian populations from the same geographical area in the United States ${ }^{7,13}$. A multiethnic study in Paris demonstrated higher prevalence of MPA in non-European populations from the Maghreb region, sub-Saharan Africa, Asia, and the Caribbean ${ }^{16}$. Differences in HLA subtypes have been investigated as a cause of global variation in the prevalence of vasculitis. HLA-DRB180401 or HLA-DRB $1 * 0404$ are commonly found associated with GCA in the United States ${ }^{1}$. Overall prevalence of these HLA haplotypes is significantly lower in healthy Japanese populations compared to healthy subjects in the United States ${ }^{1,17}$, which may explain the lower incidence of GCA in Japanese populations. Regional variation in clinical manifestations of
TA has been attributed to differences in HLA phenotype in different populations. For example, in Japan HLA-B52 is the most common HLA associated with TA, whereas HLA-B39 is more frequent in Indian and Chinese patients with $\mathrm{TA}^{1}$. HLA associations have also been reported in AAV with differing genetic associations by ANCA serotype ${ }^{9}$.

Some of the differences in prevalence and clinical variability of vasculitis in different parts of the world could be secondary to disparities in clinical practice and access to diagnostic modalities. For example, increasing use of sophisticated diagnostic modalities in developed nations may aid in early diagnosis and comprehensive phenotypic disease characterization. In the absence of these facilities, data on incidence and phenotype of vasculitis in many parts of the world may be incomplete. The relative lack of epidemiological data from specific parts of the world, most notably Africa, highlights the need for better characterization of vasculitis on a global scale.

Standardization of clinical assessment and treatment practices in observational studies and clinical trials will be required to interpret differences in clinical phenotype and outcomes across different regions of the world. Regional differences in clinical practice may be an important confounder, particularly regarding comparative studies of clinical outcomes. In both the current and previous studies by Furuta, et al, treatment strategies differed between Japan and the United Kingdom ${ }^{7,8}$. In Japan, patients with GPA typically received slower glucocorticoid tapering regimens and were less likely to receive steroid-sparing remission maintenance immunosuppressant medications compared to patients from the UK. Overall survival for patients with GPA was lower in Japan; however, relapse rates were higher in the UK cohort despite use of disease-modifying agents and biologic therapies during the remission phase of disease. Similarly, in studies of MPA, patient survival and renal survival were equivalent between patients in Europe and Japan, despite the less common use of steroid-sparing medications in the induction or remission phase of disease in the Japanese cohorts. Determining to what extent biologic factors influence clinical outcomes in different parts of the world is challenging when treatment approaches differ by region.

Ultimately, the study by Furuta, et al in this issue of The Journal demonstrates the benefit of collaborative research in rare diseases with representative partnership from different regions around the world. Future global collaborative projects in vasculitis should be prospectively standardized and highly encouraged.

\footnotetext{
SHUBHASREE BANERJEE, MD; PETER C. GRAYSON, MD, MSc,

US National Institutes of Health,

National Institute of Arthritis and Musculoskeletal and Skin Diseases (NIAMS),

Vasculitis Translational Research Program, Bethesda, Maryland, USA.
} 
Address correspondence to Dr. P.C. Grayson, National Institutes of Health, 10 Center Drive, Building 10, 6N Rm 216G, Bethesda, Maryland 20892,

USA.E-mail: peter.grayson@nih.gov

Supported through the Intramural Research Program at NIAMS.

\section{REFERENCES}

1. Kobayashi S, Fujimoto S, Takahashi K, Suzuki K. Anti-neutrophil cytoplasmic antibody-associated vasculitis, large vessel vasculitis and Kawasaki disease in Japan. Kidney Blood Press Res 2010;33:442-55.

2. Fujimoto S, Watts RA, Kobayashi S, Suzuki K, Jayne DR, Scott DG, et al. Comparison of the epidemiology of anti-neutrophil cytoplasmic antibody-associated vasculitis between Japan and the U.K. Rheumatology 2011;50:1916-20.

3. Numano F. Differences in clinical presentation and outcome in different countries for Takayasu's arteritis. Curr Opin Rheumatol 1997;9:12-5.

4. Schmidt J, Kermani TA, Bacani AK, Crowson CS, Cooper LT, Matteson EL, et al. Diagnostic features, treatment, and outcomes of Takayasu arteritis in a US cohort of 126 patients. Mayo Clin Proc 2013;88:822-30

5. Watanabe Y, Miyata T, Tanemoto K. Current clinical features of new patients with Takayasu arteritis observed from cross-country research in Japan: age and sex specificity. Circulation 2015;132:1701-9.

6. Moriwaki R, Noda M, Yajima M, Sharma BK, Numano F. Clinical manifestations of Takayasu arteritis in India and Japan - new classification of angiographic findings. Angiology 1997;48:369-79.

7. Furuta S, Chaudhry AN, Hamano Y, Fujimoto S, Nagafuchi H, Makino $\mathrm{H}$, et al. Comparison of phenotype and outcome in microscopic polyangiitis between Europe and Japan. J Rheumatol 2014;41:325-33.
8. Furuta S, Chaudhry AN, Arimura Y, Dobashi H, Fujimoto S, Homma S, et al. Comparison of the phenotype and outcome of granulomatosis with polyangiitis between UK and Japanese cohorts. J Rheumatol 2017;44:216-22.

9. Lyons PA, Rayner TF, Trivedi S, Holle JU, Watts RA, Jayne DR, et al. Genetically distinct subsets within ANCA-associated vasculitis. N Engl J Med 2012;367:214-23.

10. Specks U, Merkel PA, Seo P, Spiera R, Langford CA, Hoffman GS, et al. Efficacy of remission-induction regimens for ANCA-associated vasculitis. N Engl J Med 2013;369:417-27.

11. Gonzalez-Gay MA, Vazquez-Rodriguez TR, Lopez-Diaz MJ, Miranda-Filloy JA, Gonzalez-Juanatey C, Martin J, et al. Epidemiology of giant cell arteritis and polymyalgia rheumatica. Arthritis Rheum 2009;61:1454-61.

12. Smith CA, Fidler WJ, Pinals RS. The epidemiology of giant cell arteritis. Report of a ten-year study in Shelby County, Tennessee. Arthritis Rheum 1983;26:1214-9.

13. Huston KA, Hunder GG, Lie JT, Kennedy RH, Elveback LR. Temporal arteritis: a 25-year epidemiologic, clinical, and pathologic study. Ann Intern Med 1978;88:162-7.

14. Lane SE, Watts R, Scott DG. Epidemiology of systemic vasculitis. Curr Rheumatol Rep 2005;7:270-5.

15. Ntatsaki E, Watts RA, Scott DG. Epidemiology of ANCA-associated vasculitis. Rheum Dis Clin North Am 2010;36:447-61.

16. Mahr A, Guillevin L, Poissonnet M, Ayme S. Prevalences of polyarteritis nodosa, microscopic polyangiitis, Wegener's granulomatosis, and Churg-Strauss syndrome in a French urban multiethnic population in 2000: a capture-recapture estimate. Arthritis Rheum 2004;51:92-9.

17. Weidner N. Giant-cell vasculitides. Semin Diagn Pathol 2001; 18:24-33.

J Rheumatol 2017;44:136-9; doi:10.3899/jrheum.161468 